\title{
Role of Novel Scientific Results in Learning
}

\author{
By V.Vujnović \\ Institute of Physics of the University, P.O.Box 304, 10001 Zagreb, Croatia
}

\section{Introduction}

Introductory remarks on astronomy education in Croatia are given. Since the learning process is a complex intellectual and emotional process which should be supported during the interaction with the teacher, different approaches should be used. Tests could give useful insight into preconceptions. The following approaches should be balanced: historical approach, discovery approach (by the use of self-made tools and courtyard observations), and thorough inclusion of novel scientific results and views (to which a special precaution has been paid).

\section{The Croatian Experience}

This is a report about an experience in teaching astronomy to the students who will become teachers in physics or physics and mathematics. It should be stressed that astronomy in Croatia is not a standard subject in any schools, except as an elective course in some grammar and high schools; furthermore, astronomical concepts are partly exposed within physics.

The first step toward students should be mutual acquaintance. In order to test students' previous knowledge, I used 20-25 questions mainly of a general nature (starting in 1975). I had the opportunity to teach at all four Croatian universities: Zagreb, Osijek, Rijeka and Split. People in these towns may have different backgrounds. Zagreb is the capital of Croatia and cosmopolitan. Osijek is the center of Slavonia and belongs to an agricultural and Panonian environment. Split is heart of Dalmatia and situated on the Adriatic Sea - in the Mediterranean region. Without regard to differences in life attitudes, temperament and historical background of populations, the test showed a low level of general knowledge in natural sciencies, especially regarding comprehension of objects and scientific terms.

It is understandable that high school gives a sketchy knowledge of the universe but this knowledge is seldom useful; for example, pupils orient themselves with difficulty. They are unable to orient on the horizon (in space) and also in time by using celestial phenomena. Southern direction in a room? Local noon?

Common results of tests at the beginning of the study (knowledge obtained in the high school) can shortly be exemplified:

do not know:

tropical year

solar distance and (1975) its state of matter

meaning of zenith (confused with the superior culmination of the sun)

level of recognizability:

particular objects such as planetary nebulae

adopted concepts:

comets (object of significant interest which is restored after every apparition)

The learning process is a complex intellectual and emotional process which is influenced by a manifold of factors, including previous knowledge, self-experience, sensibility towards others' experience, and wish to comprehend the scientific views. New subject-matter can be accepted with adequate pre-knowledge, mathematical skill and basic knowledge of physical processes. Between opportunities which make the task of education easier one should look for available literature in the mother tongue, access to a planetarium, access to astronomical telescopes. Among important subjective motives there are: striving for a better mark (excellent, or very good); wish to get a diploma and to become an experienced teacher. All these factors and prerequisites have an influence on the students' learning process. And we have to help. 
In fulfilling the aim, there is no unique method or educational technology of universal application. Various approaches (interactions between teacher and learner) should be balanced. In order to stimulate students in learning, as essential I consider:

- acquaintance with the development of astronomy, and repetition of the steps of great men

- attainment of practical skills in astronomical observations

- information on the latest news and results

One can find the experience of great astronomers very stimulating since students identify with them. The historical path gives the first motivation to the students who came from the high school where they are used to learn instead of doing research. For this purpose one can use the methods applied by the great to find the proper place of the Earth in the Universe (measuring the size of the Earth with Eratosthenes, relating the distance of the moon and sun with Aristarchus, determination of the sidereal periods of the planets with Copernicus, etc). After elaborating methods in historical order, space is opened to treat particular astronomical branches in detail.

The next important step is personal research practice and attainment of practical skills. Without personal experience all scholarship loses sense. Practical work is primarily designed for orientation on the horizon (elements of positional astronomy). For the purpose a self-made apparatus could be constructed consisting of gnomon, stellar and solar altimeter (in the form of a quadrant), cross- staff and star finder. The apparatus gives results the value of which depends on the attention paid to it. It can be used to determine meridian and cardinal points in the school-yard, moments of local noon, geographical latitudes and longitudes, length of the apparent solar day, obliquity of the ecliptic. Difficulties in comprehending the relation between the real and apparent motion of the Earth and celestial bodies are overcome by the practical work which also helps in developing and adapting the concepts.

As one of the most effective stimuli in the learning process I stress the role of new or newest scientific results. Its significance can be visualizued by an easy proof of the spherical Earth: instead of spending centuries on physical and other proofs, one snapshot taken by astronauts is sufficient to eliminate all doubts. A shortcut in perception reflects in a shortcut in cognition.

How to incorporate and use novel scientific results? I will illustrate this by two examples.

a) Distance measurement of SN1987 From the tests and oral exercises I learned about poor students' understanding of the relation between the central angle and corresponding circumference. The relation between circular arc length, central angle and radius is the most simple mathematical relation - which pupils avoid since they have difficulties in comprehending the definition of the radian. By introducing the phenomenon of the recent supernova in the Large Magellanic Cloud, pointing out that it gave the first proof of neutrinos, and that it ejected a gaseous ring some 20000 years in advance of collapse - their attitude towards geometrical problems is changing. They follow as in a cartoon - paths of the light burst starting at the moment of the detected neutrino burst and directed towards the gaseous ring. They then solve simple arithmetic:

$$
s=c \cdot t=r \cdot \vartheta,
$$

where $s$ is distance travelled, $t$ time to illuminate the ring, $r$ the supernova distance and $v$ the apparent ring radius.

Students were stimulated to solve the problem and attained experience in transferring the mathematics to other analogous problems. The angular method links many astronomical affairs and is far reaching; the formal problem is the same when measuring the size of the Earth by the method of Eratosthenes, or when measuring distances by the apparent galactic diameters.

b) Planetary nebulae

For many years answers obtained during final examination about planetaries were not satisfying. Although some students could explain the evolutionary transition from red giant to white dwarf, others completely overlooked the existence of planetaries. The situation improved when an astronomy book was published in Croatian in 1989. However, qualitative change happened 
when students were really involved in the problem. We compared low-resolution images of planetaries which give an idyllic impression of simple geometry of a spherical shell leaving the rest of a star, with the higher resolution image of the Helix showing complex veils. Double bubble structure can be seen in another specimen. Deeper insight gives evidence of consecutive ejecta shown by Abell 30 (Borkowski et al,1995). This planetary shows a rounded shell of hydrogen expanding with a velocity of $40 \mathrm{~km} / \mathrm{s}$ (typical for the planetaries), ejected some 9000 years ago, and more recent ejecta from 1000 years ago, which are devoid of hydrogen and rich in carbon - thus supporting the theory of nuclear evolution of the stellar interiors. Interaction of these ejecta with the radiation and wind of the central star reveals cometary structures.

Now, the phenomenon of planetaries can be compared with other instability phenomena, as with nova or pre-supernova ejecta. By contrasting these phenomena a new intellectual relation, "feeling" about phenomena arises and with higher understanding memorization is no longer mechanical.

What has happened in the rational process? New data, laborious problems combined with the hard task of incorporating them in the previous mental attitude, engaged much more intellectual forces than simple and easy problems. The seriousness of the problem invoked new processes and students were over-engaged. To get less, better to ask for more. During this engagement, the element of surprise is found very stimulative. Not surprise only in the bare sense of the sensation present in astronomical affairs (black holes or supernovas); equally sensational is the discovery that a little calculation with clear physical insight explains equally well a pendulum-clock and pulsating stars. Analogies should be exploited as much as possible.

\section{Conclusion}

In the complex learning process many factors and prerequisites may have an influence. Incorporation of new scientific results for which an extra intellectual engagement is needed, backed by curiosity and emotional capability, may be found stimulating. This raises the quality of the interactive process between teacher and learner.

For the application of temporary scientific results, a collection of exemplary problems with methodical hints would be very useful for lecturers at any educational level. Since science frontiers proceed at a quick pace, problems should be revisited at regular intervals; since lecturers are not in a position to be "science digestors" only, effort invested by an international group would be wellcome.

\section{REFERENCES}

Borkowski, K.J., Harrington, J.P. \& Tsvetanov, Z.I. 1995, Astrophys. J., 449, L143. 